Multiple sclerosis

\section{Cortical reorganisation in patients with MS}

\section{Filippi, M A Rocca}

\section{Using functional MRI techniques to study patients with MS}

O ver the past decade, modern structural magnetic resonance imaging (MRI) techniques have been extensively used for the study of patients with multiple sclerosis (MS). ${ }^{1}$ The ultimate goal of this research is to increase the understanding of the mechanisms responsible for the accumulation of irreversible disability. Although important insight into MS pathobiology has been achieved by using modern MR based techniques, the magnitude of the correlation between MRI and clinical findings remains suboptimal. This might be explained, at least partially, by the variable effectiveness of reparative and recovery mechanisms following MS related tissue damage.

Resolution of acute inflammation, remyelination, and redistribution of voltage gated sodium channels in persistently demyelinated axons are all likely to limit the clinical impact of damaging MS pathology. ${ }^{2}$ Cortical reorganisation has been recently suggested, however, as an additional potential contributor to the recovery or to the maintenance of function in the presence of irreversible MS related tissue damage. This is in agreement with what has been seen in other neurological conditions, such as stroke, ${ }^{3}$ tumours, ${ }^{45}$ and Alzheimer's disease. ${ }^{67}$ Brain plasticity is indeed a well known feature of the human brain, which is likely to have several different substrates (including increased axonal expression of sodium channels, ${ }^{8}$ synaptic changes, increased recruitment of parallel existing pathways or "latent" connections, and reorganisation of distant sites), and which might have a major adaptive role in limiting the functional consequences of axonal loss in MS. ${ }^{9}$

\section{What is measured using fMRI?}

The signal changes seen during functional MRI (fMRI) studies depend on the blood oxygenation level dependent mechanism, which, in turn, involves changes of the transverse magnetisation relaxation time-either $\mathrm{T} 2{ }^{*}$ in a gradient echo sequence or $\mathrm{T} 2$ in spin echo sequence. ${ }^{5}$ Local increases in neuronal activity result in a rise of blood flow and oxygen consumption. The increase of blood flow is greater than the oxygen consumption thus determining an increased ratio between oxygenated and deoxygenated haemoglobin, which enhances the MRI signal. ${ }^{10}$ As these signal changes are very modest (usually ranging from $0.5-1.5 \%$ ), it is usually necessary to obtain a large amount of brain images acquired during alternated periods of activations (motor, sensorial, and cognitive) and rest. As a consequence, concern has been raised regarding inter-subject and inter-session variability in fMRI signal. However, by analysing these data with appropriate statistical methods, it is possible to obtain information about the location and the extent of specific areas involved in the performance of a given task in healthy subjects and in patients with different neurological conditions. ${ }^{11}$

One of the main problems in the interpretation of clinical fMRI studies is that the observed changes might be influenced by differences in task performance between patients and controls. Clearly this is a major issue in MS, which typically causes impairment of various functional systems. To overcome this problem fMRI studies of MS have either selected patients with no overt clinical symptomatology of the investigated functional systems ${ }^{12-21}$ or, in case of motor tasks, passive movements have been used as the experimental paradigm..$^{22}$

\section{Does fMRI disclose abnormal patterns of cortical activations in patients with MS?}

An altered recruitment of regions normally devoted to the performance of a given task and/or the recruitment of additional areas that are not typically activated by healthy people for performing that given task have been described in patients with MS, independent of their clinical phenotype, when investigating the visual, ${ }^{2324}$ cognitive, $^{12} 132526$ and motor ${ }^{12-22} 27-32$ systems. The following items summarise the main findings supporting this notion:
- Increased recruitment of task related brain regions have been observed in patients at presentation with clinically isolated syndromes suggestive of MS during the performance of visual $^{24}$ and motor ${ }^{17}{ }^{20}$ tasks. This agrees with data obtained from subjects at risk of developing Alzheimer's disease, ${ }^{7}$ and suggests the potential of fMRI for detecting an abnormal pattern of cortical activations early in the course of various brain pathologies.

- Increased recruitment of task related brain regions has also been detected in patients with early relapsing remitting (RR) $\mathrm{MS}^{30}{ }^{31}$ in those with RRMS and no residual clinical disability, ${ }^{16}$ and in RRMS patients with mild to moderate disability ${ }^{12} 25-28$ during the performance of motor $^{1627283031}$ and cognitive $e^{122526}$ tasks.

- Increased recruitment of motor related brain regions has also been described in patients with primary progressive $(\mathrm{PP})^{15} 32$ and secondary progressive (SP) ${ }^{18} 2728$ MS with different levels of motor impairment and clinical disability.

Data on patients affected by stroke ${ }^{3}$ seem to indicate that recovery is enhanced when a restoration of activation towards the physiological network is observed over time. This might not be the case of MS, as suggested by the available cross-sectional studies, ${ }^{12-32}$ because new lesion formation and accumulating microscopic damage in central nervous system areas variably related to those already damaged have the potential to influence the final outcome of cortical reorganisation.

\section{Is the extent of functional cortical reorganisation correlated to the extent of brain damage in MS?}

Several studies based on quantitative MR techniques (such as magnetisation transfer (MT) MRI, diffusion weighted (DW) MRI, and MR spectroscopy) have shown that MS damage is not limited to macroscopic visible lesions, in which different degrees of tissue loss/disruption can be detected, but also involves extensively the normal appearing white matter and normal appearing grey matter. ${ }^{1}$ Recent work has shown that functional and structural changes of the MS brain are strictly correlated, as detailed in the following items:

- An increased cortical recruitment with increasing T2 lesion load has been shown in patients with relapsing $^{162731}$ and $\mathrm{PP}^{32}$ MS.

- The severity of intrinsic T2 visible lesion damage, measured using MT 
and DW MRI, has been found to modulate the activity of some cortical areas. An increased recruitment of the primary sensorimotor cortex has been shown to be correlated with the severity of lesion damage of the corticospinal tracts. This is measured using Tl weighted images ${ }^{31}$ and with the whole brain average lesion MT ratio and mean diffusivity. ${ }^{16}$

- The severity of normal appearing brain tissue damage, measured using MR spectroscopy, ${ }^{17}$ MT MRI, and DW MRI, ${ }^{15} 1618$ is another important factor modulating increased recruitment of motor related brain regions. This is shown by studies of patients at presentation with clinically isolated syndrome suggestive of $\mathrm{MS}^{17}$ patients with RRMS and variable degrees of clinical disability, ${ }^{16}{ }^{28}$ and patients with PPMS and SPMS. ${ }^{15} 18$ Similar findings have been also obtained from patients with migraine $^{33}$ and cerebral autosomal dominant arteriopathy with subcortical infarcts and leukoencephalopathy (CADASIL). ${ }^{34}$

- Subtle grey matter damage, which goes undetected when using conventional MRI, may also influence functional cortical recruitment. This is demonstrated in patients with SPMS $^{18}$ and in patients with clinically definite MS and non-specific (less than three lesions) conventional MRI findings. ${ }^{19}$

\section{Is the extent of functional cortical reorganisation in $\mathrm{MS}$ related to brain damage only?}

Two structures that are frequently affected by MS pathology, and whose involvement might contribute to explain MS related disability, are the spinal cord and the optic nerve. FMRI studies, which investigated the role of the involvement of these structures on cortical reorganisation, have shown that:

- Cord pathology can induce cortical changes with the potential to limit the functional impact of the disease. This is demonstrated in patients with PPMS, ${ }^{15}$ where a strong correlation between the extent of the fMRI activations of several sensorimotor areas and several MT MR metrics of structural damage of the cervical cord has been found.

- Brain adaptive cortical changes can be elicited by cord pathology. This is shown in patients with a previous episode of isolated acute myelitis of probable demyelinating origin and lack of brain damage ${ }^{35}$ and in patients with neuromyelitis optica. ${ }^{36}$
In both these conditions an increased activation of several cortical areas, mainly located in the ipsilateral hemisphere, was demonstrated during the investigation of a simple motor task performed with the dominant, clinically unaffected, upper limb. The strong relationship found between fMRI activations and MT MRI metrics of cervical cord damage suggests that such changes might be adaptive.

- A decreased activation of the primary visual cortex, ${ }^{23}{ }^{37}$ and an increased recruitment of secondary visual areas, $^{24}$ has also been detected in patients with previous optic neuritis (ON). Unfortunately, because of technical limitations of MT and DT MRI in the study of the ON, none of these authors has investigated the relationship between the severity of ON damage and the extent of fMRI activations. However, the demonstration of a different pattern of activation according to the latency of the visual evoked potentials ${ }^{24}$ suggests that, as shown for the cord, ${ }^{15} 3536$ the severity of ON involvement also might influence the brain pattern of cortical activation.

\section{Which is the role of functional cortical reorganisation in MS?}

The main hypothesis that prompted the extensive application of fMRI to the assessment of MS suggested that functional cortical reorganisation might play a role in limiting the impact of structural tissue damage in MS patients and, conversely, that its progressive exhaustion with disease progression might be one of the factors contributing to the accumulation of irreversible disability. Although the actual role of cortical reorganisation on the clinical manifestations of MS remains unclear, there are several pieces of evidence, in addition to the strong correlation found between functional and structural changes, that suggest that cortical adaptive changes are likely to contribute in limiting the clinical consequences of MS related structural damage: ${ }^{9}$

- As previously shown in patients with stroke affecting the motor system, ${ }^{3}$ in a patient with an acute hemiparesis following a new, large demyelinating lesion, located in the corticospinal tract, dynamic changes of the brain pattern of activation of the "classical" motor areas, ending in a full recovery of function, have been observed. ${ }^{29}$ The correlation found between the extent of functional cortical changes and $N$ acetylaspartate levels suggests that dynamic reorganisation of the motor cortex can occur in response to axonal injury associated with MS activity.

- A reduced activation of a complex movement associated cortical/subcortical network, including the cerebellum, the rolandic operculum, the thalamus, and the middle frontal gyrus, has been found in patients complaining of fatigue when compared with matched non-fatigued MS patients. ${ }^{14}$ In fatigued patients a strong correlation between the reduction of thalamic activity and the clinical severity of fatigue was also found, indicating that a less marked cortical recruitment might be associated to the appearance of clinical symptomathology in MS.

- A recent study has provided a direct demonstration that MS patients during the performance of a simple motor task tend to activate regions that are usually activated by healthy subjects during the performance of more complex tasks, such as regions, that are part of a frontoparietal circuit, whose activation occurs typically in healthy subjects during object manipulation. ${ }^{21}$

- Preliminary work has shown that the pattern of movement associated cortical activations in MS is determined by both the extent of brain injury and disability and that these changes are distinct. $^{22}$

\section{Does $f M R I$ cortical reorganisation evolve during the course of the disease?}

As shown in patients affected by stroke, ${ }^{3}$ dynamic functional changes have been also described in a MS patient following an acute relapse. ${ }^{29}$ More recently, it has been postulated that dynamic changes of brain cortical activations might occur with the progression of the disease from the RR to the SP phase. However, currently no longitudinal fMRI studies have been conducted in MS patients and no direct comparison of the pattern of cortical activation between MS patients with different disease phenotypes has been performed. In addition, the majority of the fMRI studies were focused on the motor system of MS patients and there are only a few reports on the visual and cognitive systems. Nevertheless, data from available studies ${ }^{15-18} 20-223032$ suggest that the functional reorganisation of the cerebral cortex in MS patients might change over the course of the disease. At the early stages an increased recruitment of those areas "normally" devoted to the performance of a given task, such as the primary sensorimotor cortex in the case of a motor task, ${ }^{17} 20$ is likely to occur. At a 
later stage, bilateral activation of these regions might first be seen ${ }^{30}$ followed, with progressive disability accumulation, by a widespread recruitment of additional areas, which are usually recruited in normal people to perform novel/complex tasks. ${ }^{15} 1618212232$

\section{CONCLUSION}

FMRI has the potential to provide important pieces of information about cortical reorganisation following MS tissue damage, which should improve our understanding of the factors associated to the progressive accumulation of irreversible disability in MS. Although the role of cortical reorganisation in limiting the functional impact of MS structural damage is still not definitively proved, the available data support the concept that cortical adaptive responses may have a role in compensating for tissue damage in MS. They also suggest that the rate of accumulation of disability in MS might not only be a function of tissue loss, but also of progressive failure of adaptive capacity of the cortex. In healthy subjects, plastic adaptive changes within the motor network have been implicated in the maintenance of a normal motor performance with ageing. ${ }^{38}$ In MS patients, the lack or the progressive exhaustion of the "classical" adaptive mechanisms and the need of "second order" compensatory areas might be among the factors contributing to the accumulation of irreversible clinical deficits. The enhancement of any beneficial effects of this cortical adaptive plasticity should be considered as a potential target of therapy for MS.

J Neurol Neurosurg Psychiatry 2004;75: 1087-1089

doi: 10.1136/jnnp.2004.036020

\section{Authors' affiliations}

M Filippi, M A Rocca, Neuroimaging Research Unit, Department of Neurology, Scientific Institute and University Ospedale San Raffaele, Milan, Italy

\section{Correspondence to: Dr M Filippi,} Neuroimaging Research Unit, Department of Neurology, Scientific Institute and University Ospedale San Raffaele, via Olgettina 60, 20132 Milan, Italy; filippi.massimo@hsr.it

This study was supported by a grant from Fondazione Italiana Sclerosi Multipla (FISM) 2002/R/28)

Competing interests: none declared

\section{REFERENCES}

1 Filippi M, Rocca MA, Comi G. The use of quantitative magnetic-resonance-based techniques to monitor the evolution of multiple sclerosis. Lancet Neurol 2003;2:337-46.

2 Waxman SG, Ritchie JM. Molecular dissection of the myelinated axon. Ann Neurol 1993:33:121-36.

3 Calautti C, Baron JC. Functional neuroimaging studies of motor recovery after stroke in adults: a review. Stroke 2003;34:1553-66.

4 Holodny Al, Schulder M, Ybasco A, et al. Translocation of Broca's area to the contralateral hemisphere as the result of the growth of a left inferior frontal glioma. J Comput Assist Tomogr 2002; 26:941-3.

5 Carpentier AC, Constable RT, Schlosser MJ, et al. Patterns of functional magnetic resonance imaging activation in association with structural lesions in the rolandic region: a classification system. J Neurosurg 2001;94:946-54.

6 Machulda MM, Ward HA, Borowski B, et al. Comparison of memory FMRI response among normal, $\mathrm{MCl}$, and Alzheimer's patients. Neurology 2003;61:500-6.

7 Bookheimer SY, Strojwas MH, Cohen MS, et al. Patterns of brain activation in people at risk for Alzheimer's disease. N Engl J Med 2000;343:450-6.

8 Waxman SG. Demyelinating diseases: New pathological insights, new therapeutic targets. New Engl J Med 1998;338:323-6.

9 Filippi M, Rocca, MA. Disturbed function and plasticity in multiple sclerosis as gleaned from functional magnetic resonance imaging. Curr Opin Neurol 2003;16:275-82.

10 Ogawa S, Menon RS, Tank DW, et al. Functional brain mapping by blood oxygenation leveldependent contrast magnetic resonance imaging. A comparison of signal characteristics with a biophysical model. Biophys J 1993;64:803-12.

11 Matthews PM, Jezzard P. Functional magnetic resonance imaging. J Neurol Neurosurg Psychiatry 2004;75:6-12.

12 Staffen W, Mair A, Zauner H, et al. Cognitive function and FMRI in patients with multiple sclerosis: evidence for compensatory cortical activation during an attention task. Brain 2002; 156: 1275-82.

13 Hillary FG, Chiaravalloti ND, Ricker JH, et al. An investigation of working memory rehearsal in multiple sclerosis using fMRI. J Clin Exp Neuropsychol 2003;25:965-78.

14 Filippi M, Rocca MA, Colombo B, et al. Functional magnetic resonance imaging correlates of fatigue in multiple sclerosis. Neurolmage 2002; 15:559-67.

15 Filippi M, Rocca MA, Falini A, et al. Correlations between structural CNS damage and functional MRI changes in primary progressive MS Neurolmage 2002;15:537-46.

16 Rocca MA, Falini A, Colombo B, et al. Adaptive functional changes in the cerebral cortex of patients with non-disabling MS correlate with the extent of brain structural damage. Ann Neurol 2002;51:330-9.

17 Rocca MA, Mezzapesa DM, Falini A, et al. Evidence for axonal pathology and adaptive cortical reorganisation in patients at presentation with clinically isolated syndromes suggestive of MS. Neurolmage 2003;18:847-55

18 Rocca MA, Gavazzi C, Mezzapesa DM, et al. A functional magnetic resonance imaging study of patients with secondary progressive multiple sclerosis. Neurolmage 2003;19:1770-7.

19 Rocca MA, Pagani E, Ghezzi A, et al. Functional cortical changes in patients with MS and nonspecific conventional MRI scans of the brain. Neurolmage 2003;19:826-36.
20 Filippi M, Rocca MA, Mezzapesa DM, et al. Simple and complex movement-associated functional MRI changes in patients at presentation with clinically isolated syndromes suggestive of MS. Human Brain Mapping 2004;21:108-17.

21 Filippi M, Rocca MA, Mezzapesa DM, et al. A functional MRI study of cortical activations associated with object manipulation in patients with MS. Neurolmage 2004;21:1147-54.

22 Reddy H, Narayanan S, Woolrich $M$, et al. Functional brain reorganisation for hand movement in patients with multiple sclerosis: defining distinct effects of injury and disability. Brain 2002;125:2646-57.

23 Rombouts SA, Lazeron RH, Scheltens P, et al Visual activation patterns in patients with optic neuritis: an FMRI pilot study. Neurology 1998;50:1896-9.

24 Werring DJ, Bullmore ET, Toosy AT, et al. Recovery from optic neuritis is associated with a change in the distribution of cerebral response to visual stimulation: a functional magnetic resonance imaging study. J Neurol Neurosurg Psychiatry 2000;68:441-9.

25 Parry AM, Scott RB, Palace J, et al. Potentially adaptive functional changes in cognitive processing for patients with multiple sclerosis and their acute modulation by rivastigmine. Brain 2003; 126:2750-60.

26 Wishart HA, Saykin AJ, McDonald BC, et al. Brain activation patterns associated with working memory in relapsing-remitting MS. Neurology 2004:62:234-8.

27 Lee $M$, Reddy $\mathrm{H}$, Johansen-Berg $\mathrm{H}$, et al. The motor cortex shows adaptive functional changes to brain injury from multiple sclerosis. Ann Neurol 2000;47:606-13.

28 Reddy H, Narayanan S, Arnoutelis R, et al. Evidence for adaptive functional changes in the cerebral cortex with axonal injury from multiple sclerosis. Brain 2000;123:2314-20.

29 Reddy H, Narayanan S, Matthews PM, et al. Relating axonal injury to functional recovery in MS. Neurology 2000;54:236-9.

30 Pantano P, lannetti GD, Caramia F, et al. Cortical motor reorganisation after a single clinical attack of multiple sclerosis. Brain 2002;125:1607-15.

31 Pantano P, Mainero C, lannetti GD, et al. Contribution of corticospinal tract damage to cortical motor reorganisation after a single clinical attack of multiple sclerosis. Neurolmage 2002; 17:1837-43.

32 Rocca MA, Matthews PM, Caputo D, et al. Evidence for widespread movement-associated functional MRI changes in patients with PPMS Neurology 2002;58:866-72.

33 Rocca MA, Colombo B, Pagani E, et al. Evidence for cortical functional changes in patients with migraine and white matter abnormalities on conventional and diffusion tensor magnetic resonance imaging. Stroke 2003;34:665-70.

34 Reddy H, De Stefano N, Mortilla M, et al. Functional reorganisation of motor cortex increases with greater axonal injury from CADASIL. Stroke 2002;33:502-8.

35 Rocca MA, Mezzapesa DM, Ghezzi A, et al. Cord damage elicits brain functional reorganisation after a single episode of myelitis. Neurology 2003:61:1078-85.

36 Rocca MA, Agosta F, Mezzapesa DM, et al. A functional MRI study of movement-associated cortical changes in patients with Devic's neuromyelitis optica. Neurolmage 2004;1:1061-8.

37 Langkilde AR, Frederiksen JL, Rostrup E, et al. Functional MRI of the visual cortex and visual testing in patients with previous optic neuritis. Eur J Neurol 2002;9:277-86.

38 Ward NS, Frackowiak RSJ. Age-related changes in the neuronal correlates of motor performance. Brain 2002; 126:873-8. 
(a) classical presentations without onconeural antibodies or identified cancer but with high risk of an underlying

\section{Recommended diagnostic criteria for paraneoplastic neurological syndromes}

\section{J E Greenlee}

neoplasia; (b) a neurological syndrome (classical or otherwise) with partially characterised onconeural antibodies and no cancer; and (c) a non-classical neurological condition without onconeural antibodies, but with cancer identified within 2 years of neurological presentation.

Graus and his colleagues have performed a significant service for neurologists by providing a system for assessing whether or not a given patient has a paraneoplastic neurological disorder. The criteria that they have developed should allow categorisation of newly identified paraneoplastic syndromes and, of even greater importance, should be of real benefit in standardising both research protocols and multiinstitutional therapeutic trials.

J Neurol Neurosurg Psychiatry 2004;75:1090. doi: 10.1136/jnnp.2004.038489 been that many affected patients have serum and cerebrospinal fluid (CSF) antibodies that are reactive with internal neuronal antigens. ${ }^{12}$ In some cases, there is strong correlation between patterns of antibody reactivity and specific tumours, and tumours from some affected patients have been found to express proteins that immunologically crossreact with neuronal proteins. ${ }^{3}{ }^{4}$ Thus, detection of antineuronal autoantibodies in a patient with a progressive neurological disorder may allow the diagnosis of a paraneoplastic syndrome and also provide information about the nature of the underlying neoplasm. ${ }^{124}$

The major paraneoplastic neurological syndromes had been largely categorised by 1965 , and this same categorisation is still used for diagnosis today, supported by detection of antineuronal autoantibodies. In some instances, however, determination as to whether a given patient has a paraneoplastic syndrome may prove difficult. Firstly, not all patients with paraneoplastic neurological syndromes have antineuronal

antibodies, nor do all patients with antineuronal antibodies have paraneoplastic neurological syndror in whom no tumour can be detect and high titres of antineuronal antibodies. Finally, uniform diagnostic criteria for paraneoplastic neurological syndromes, incorporating both clinical and serological data, have not been established, nor is there an established framework for categorisation of newly discovered syndromes. This same lack of standardised criteria for diagnosis of these disorders also hinders development of collaborative therapeutic trials.

Graus et al, in their paper on page $1135,{ }^{5}$ present criteria by which patients with suspected paraneoplastic syndromes can be divided into "definite" and "probable" categories, based upon immunological and clinical data. "Definite" syndromes include (a) classical presentations (such as cerebellar degeneration or encephalomyeloneuritis) accompanied by cancer developing within 5 years of diagnosis of the neurological disorder; $(b)$ a non-classical presentation that remits following cancer treatment without concomitant immunotherapy; (c) non-classical presentations with onconeural antibodies and cancer developing within 5 years of neurological diagnosis; and $(d)$ a neurological disorder, classical or otherwise, in which well characterised onconeural antibodies are present but no cancer can be found. "Possible" syndromes include
Correspondence to: Dr J E Greenlee, Veterans' Affairs Medical Center and Department of

Neurology, University of Utah Health Sciences Center, Salt Lake City, UT, USA; igreenlee@ pol.net

Competing interests: none declared

\section{REFERENCES}

1 Darnell RB, Posner JB. Paraneoplastic syndromes involving the nervous system. N Engl J Med 2003;349: 1543-54

2 Moll JW, Antoine JC, Brashear HR, et al. Guidelines on the detection of paraneoplastic anti-neuronal-specific antibodies: report from the Workshop to the Fourth Meeting of the International Society of Neuro-Immunology on Paraneoplastic Neurological Disease, October 22-23, 1994, Rotterdam, The Netherlands. Neurology 1995;45:1937-41.

3 Furneaux HM, Rosenblum MK, Dalmau J, et al. Selective expression of Purkinje cell antigens in tumor tissue from patients with paraneoplastic cerebellar degeneration. N Engl J Med 1990:322:1844-51.

1990;322: 1844-51. windows into neuronal function and tumor immunity. Arch Neurol 2004;61:30-2.

5 Graus FR, Delattre J-Y, Antoine JC, et al. Recommended diagnostic criteria for paraneoplastic neurological syndromes. J Neurol Neurosurg Psychiatry 2004;75:1135-40. 